\title{
RÉVOLUTION INDUSTRIELLE LOGIQUE ET SIGNIFICATION DE L'OPÉRATOIRE
}

\author{
Marie-José DuRAnd-Richard
}

RÉSumé : Dans la première moitié du XIX $x^{\mathrm{e}}$ siècle en Angleterre, autour de Charles Babbage (1791-1871), John F. W. Herschel (1792-1871), George Peacock (17911858), Duncan F. Gregory (1813-1844), Augustus de Morgan (1806-1871), George Boole (1815-1864), et d'autres auteurs moins connus, un réseau d'algébristes renouvelle singulièrement la conception de l'algèbre, à tel point que leur travail est le plus souvent interprété comme émergence des travaux sur l'algèbre abstraite. Comme ces algébristes sont également des réformateurs impliqués dans la réorganisation de la science, il s'agira de proposer de ces travaux une lecture plus contextuelle, afin d'éclairer leur inscription parmi les effets de la Révolution industrielle (1760-1830), et d'analyser leur rôle de médiation culturelle entre les racines d'un savoir universitaire fondé sur la fidélité aux Anciens, et de nouvelles formes d'élaboration des connaissances. Une médiation marquée par un premier rapprochement entre algèbre et logique.

Mots-CLés : algèbre, logique, Révolution industrielle.

ABSTRACT : In England, during the first half of the XIX ${ }^{\text {th }}$ century, a network organised around Charles Babbage (1791-1871), John F. W. Herschel (1792-1871), George Peacock (1791-1858), Duncan F. Gregory (1813-1844), Augustus de Morgan (1806-1871), George Boole (1815-1864), and other less known authors renewed the status of algebra in a peculiar way. As a result, their works are often considered to mark the birth of abstract algebra. But these algebraists were also involved, as reformers, in the new organisation of science. Taking this into account, I propose a more contextual reading of their works, which can then be understood as side-effects of the Industrial Revolution (1760-1830). These works are analysed as cultural mediators between the roots of University teaching, founded on fidelity to the Ancients on the one hand, and new forms of construction of knowledge on the other. Such a mediation brought together algebra and logic for the first time.

KEYWORDS : algebra, logic, Industrial Revolution.

Revue de synthèse : $4^{\mathrm{e}}$ sér., ${ }^{\text {os }}$ 2-3-4, avr.-déc. 2001, p. 319-346. 
ZuSAMmENFASSUNG : Während der ersten Hälfte des 19. Jahrhunderts in England erneuert ein Netzwerk von Algebraikern um Charles Babbage (1791-1871), John F. W. Herschel (1792-1871), George Peacock (1791-1858), Duncan F. Gregory (1813-1844), Augustus de Morgan (1806-1871), George Boole (1815-1864), und andere weniger bekannte Autoren, auf außerordentliche Weise die Konzeption der Algebra so sehr, daß ihre Arbeiten meist als der Beginn der Arbeiten zur abstrakten Algebra interpretiert werden. Da diese Algebraiker gleichzeitig Reformatoren sind, die sich für die Reorganisation der Wissenschaft einsetzen, wäre es angebracht für diese Arbeiten eine kontextuellere Lektüre vorzuschlagen, um ihre Einbettung in die Auswirkungen der Industriellen Revolution (1760-1830) zu erläutern, und ihre Rolle in der kulturellen Vermittlung zwischen den Wurzeln eines universitären Wissens, das auf der Treue zu den Alten beruht, und den neuen Formen der Wissenserarbeitung zu analysieren. Diese Art der Vermittlung ist gekennzeichnet durch eine erste Annäherung von Algebra und Logik.

STICHWÖRTER : Algebra, Logik, Industrielle Revolution.

Marie-José DuRAND-Richard, née en 1944, est maître de conférences en histoire et en épistémologie des sciences et des techniques à l'université de Paris 8-Vincennes-Saint-Denis, et chercheur associé de l'équipe REHSEIS (UMR 7596 CNRS-Paris 7). Son travail porte essentiellement sur l'étude de l'émergence et du rôle historique effectif de l'approche symbolique de l'algèbre en Angleterre, au sortir de la Révolution industrielle, une approche qui marque aussi bien les avancées d'un Charles Babbage (1791-1871) sur les fonctions logiques d'un calculateur, que celles d'un George Boole (1815-1864) sur l'algèbre de la logique. Ses orientations présentes ont pour objet la question du lieu de la signification qui, depuis la coupure entre calcul symbolique et interprétation, ne cesse de ressurgir, aussi bien en logique qu'en mathématiques, et jusqu'aux plus récents développements de l'Intelligence Artificielle.

Adresse : REHSEIS (CNRS, Université Paris 7), Centre Javelot, 2 pl. Jussieu, F-75251 Paris Cedex 05.

Courrier électronique : mjdurand@paris7.jussieu.fr

Web : http://www.ai.univ-paris8.fr/maths.somat/htm 
Cet article se veut un bilan de recherche ${ }^{1}$ sur les travaux des algébristes anglais de la première moitié du $\mathrm{XIX}^{\mathrm{e}}$ siècle, usuellement crédités de l'introduction de la notation leibnizienne du calcul infinitésimal dans les examens à l'université de Cambridge, de l'élaboration d'une conception essentiellement symbolique de l'algèbre, et de la production de nouveaux objets mathématiques. Mon propos est, plus spécifiquement, d'examiner les conditions épistémologiques et sociologiques d'élaboration de cette nouvelle approche de l'algèbre, et de pointer leur communauté de perspectives. Après avoir indiqué les limitations du questionnement inhérentes à une lecture récurrente de l'histoire des mathématiques, je montrerai comment la nouveauté de cette conception de l'algèbre peut être située comme une réponse aux débats qui agitent alors le monde savant en Grande-Bretagne, et qui portent à la fois sur le rôle des universités anglicanes anglaises de Cambridge et d'Oxford au sortir de la Révolution industrielle (1760-1830), sur la nature des fondements de la connaissance et sur la part respective de l'induction et de la déduction dans le raisonnement scientifique. Ce faisant, l'apport de ces mathématiciens apparaîtra comme ouvrant d'autres voies de recherche que celles qui leur sont traditionnellement attribuées.

\section{VERS UNE LECTURE HERMÉNEUTIQUE DE CE MOMENT D'HISTOIRE DES MATHÉMATIQUES}

Ces algébristes anglais ne sont pas seulement ceux de la liste fournie par Lubos Novy $^{2}$ : Charles Babbage ${ }^{3}$, George Peacock, Augustus de Morgan et Duncan F. Gregory, auxquels il rattache également George Boole et William R. Hamilton, ainsi que Arthur Cayley et James J. Sylvester. John F. W. Herschel en fait également partie, non seulement parce qu'il est cotraducteur en 1816, avec Babbage et Peacock, du traité qui marque l'introduc-

1. Ces recherches ont pris corps grâce à la rencontre et au soutien initial de MM. Charles Morazé et Ernest Coumet, dont l'ouverture de vue m'a permis d'échapper aux querelles souvent reconduites entre les approches internaliste et externaliste de l'histoire des mathématiques.

2. Novy, 1968.

3. Charles Babbage (1791-1871), George Peacock (1791-1858), Augustus de Morgan (1806-1871), Duncan Farquharson Gregory (1813-1844), George Boole (1815-1844), William Rowan Hamilton (1805-1865), Arthur Cayley (1821-1895), James Joseph Sylvester (18141897), John F. W. Herschel (1792-1871). 
tion de la notation leibnizienne du calcul infinitésimal dans l'enseignement à Cambridge, le Traité élémentaire de calcul différentiel et intégral (1802) de Sylvestre F. Lacroix, mais surtout parce qu'il est, dès cette même date, le premier à appliquer la méthode de séparation des variables de Louis F. A. Arbogast ${ }^{4}$ à la résolution des équations différentielles ${ }^{5}$, et qu'il est l'auteur d'un théorème important sur le calcul des opérations, reconnu comme tel par Hamilton ${ }^{6}$ dès 1828. Il faut leur adjoindre une seconde génération d'algébristes ${ }^{7}$, peu connus pour la plupart: Robert Murphy, Robert Ellis, S. S. Greatheed, Philip Kelland, John Thomas Graves, Charles Graves, Brice Bronwin, William Donkin, Robert Carmichaël, John Hewitt Jellett, Charles Hargreave, John R. Young, William Spottiswoode. Mieux vaudrait donc parler d'un réseau d'algébristes anglais ${ }^{8}$, pour souligner le fait que leur situation n'est pas celle de disciples prolongeant la pensée d'un maître installé institutionnellement, mais bien plutôt celle d'un groupe partageant certains principes généraux quant à la nature de l'algèbre. Cette dénomination prolonge également la problématique de l'historien des sciences Walter F. Cannon, qui situe Babbage, Herschel et Peacock au cœur d'un vaste réseau de réformateurs, le «network of Cambridge », proche du Broad Church Movement et des Whigs, voire, pour certains, des radicaux ${ }^{9}$.

\section{Les insuffisances d'une présentation récurrente}

Pour bon nombre d'ouvrages de référence, l'approche rétrohistorique reste de rigueur, avec la préoccupation essentielle d'apercevoir ce qui est moderne dans les travaux du passé, et de répertorier les précurseurs des théories ultérieures, cela bien que le recours à la notion de précurseur ait déjà été fortement critiquée par Georges Canguilhem.

\footnotetext{
4. Louis François Antoine Arbogast (1759-1803).

5. Ce que lui reconnaît Gregory, 1839b. Voir Arbogast, 1800.

6. $\mathrm{f}(1+\Delta) \mathrm{u}_{\mathrm{x}}=\mathrm{f}\left(\mathrm{e}^{\Delta \mathrm{x} . \mathrm{D}}\right) \mathrm{u}_{\mathrm{x}}$. Graves, 1882, vol. I, p. 304-305, lettre de Hamilton à Herschel du $28 / 10 / 1828$.

7. Ils sont presque tous diplômés d'un Trinity College, à Cambridge ou à Dublin, mais ne deviendront pas tous des mathématiciens professionnels. Les dates qui manquent aux auteurs de la liste ci-dessous n'ont été trouvées dans aucun document de référence : Robert Murphy (1806-1843), Robert Ellis (1817-1859), S. S. Greatheed, Philip Kelland (1808-1879), John Thomas Graves (1806-1870), Charles Graves (1812-1899), Brice Bronwin, William Donkin (1814-1869), Robert Carmichaël, John Hewitt Jellett (1817-1888), Charles Hargreave (18201866), John R. Young, William Spottiswoode (1825-1883).

8. Sur ce point, je suis une remarque que me fit Ivor Grattan-Guinness, concernant la distinction à faire entre «école » et « réseau », à Wuhan (Chine), en octobre 1998, au colloque international «Transmission and transformation of mathematical thought. A comparative approach ».

9. CANNON, 1964.
} 
Nicolas Bourbaki salue les algébristes de l'école anglaise comme ceux qui :

«[...] entre 1830 et 1850 , réalisent, parallèlement aux travaux de Galois et de Gauss, les progrès les plus nets vers l'abstraction, dégageant la notion abstraite de loi de composition à partir de réflexions sur la nature des nombres imaginaires, et élargissant immédiatement le champ de l'algèbre en appliquant cette notion à une foule d'êtres mathématiques nouveaux: algèbre de la logique avec Boole, vecteurs, quaternions et systèmes hypercomplexes généraux avec Hamilton, matrices et lois non associatives avec Cayley ${ }^{10}$. »

Première remarque : bon nombre des auteurs cités par Novy ne sont pas nommés par Bourbaki, notamment Peacock, qui n'a effectivement pas produit d'êtres mathématiques nouveaux, mais qui représente cependant une référence importante pour ses contemporains algébristes ${ }^{11}$. Et lorsqu'il est cité par des ouvrages anglo-saxons, comme dans celui de Carl B. Boyer, qui le traite pompeusement $d^{\prime}$ ' «Euclide de l'algèbre ${ }^{12}$ », leurs auteurs tantôt minimisent son rôle ${ }^{13}$, tantôt considèrent qu'il appréhende l'algèbre comme étude de structures abstraites ${ }^{14}$.

Il est vrai que la lecture de Peacock au seul crible de nos connaissances présentes peut induire une interprétation beaucoup plus moderne que celle qui tiendra compte de la signification contextuelle de son projet. Dès l'introduction, en 1833, de son « Report on the recent progress and actual state of certain branches of analysis », Peacock présente en effet l'algèbre comme une science purement «spéculative», comme le «langage du raisonnement symbolique », et comme science des «combinaisons de symboles arbitraires », dont la « consistance» n'est garantie que par un raisonnement déductif fondé sur des «principes premiers considérés comme seuls faits ultimes $»^{15}$.

\section{Les problèmes qu'une telle lecture laisse en suspens}

Mais cette lecture de Peacock comme première tentative d'axiomatisation de l'algèbre se heurte à des questions qu'elle laisse sans réponse :

\footnotetext{
10. Bourbaki, 1969, p. 74.

11. Hamilton le cite abondamment en 1853 dans ses Lectures on quaternions, le reconnaît pour s'en démarquer en 1837, dans sa « Theory of conjugate functions », et s'inquiète de son point de vue dès 1828, voir Hamilton, 1853, 1837; Hankins, 1980. S'y réfèrent également Kelland, 1840, et Gregory, 1840. Boole se réfère, quant à lui, à Peacock en 1844 et à l'Algèbre Symbolique en 1847.

12. BOYER, 1968.

13. Clock, 1964, p. 13-26; Koppelman, 1969, p. 75-77.

14. KLINE, 1972, p. 730.

15. Реaсоск, 1833, p. 194-200.
} 
- pourquoi un mathématicien dont l'érudition est reconnue par tous ses contemporains veut-il fonder le calcul différentiel sur le développement d'une fonction en série de Taylor, sans recourir à la notion de limite, et cela dix ans après la contribution d'Augustin Louis Cauchy (1789-1857) sur la question, un auteur qu'il reconnaît lui-même comme un brillant analyste?

- pourquoi refuse-t-il le distinguo établi par ce même Cauchy, et repris par Niels H. Abel (1802-1829), entre séries convergentes et séries divergentes?

- pourquoi ses successeurs, surtout Morgan et Gregory, le suivront-ils sur cette voie?

- pourquoi refuse-t-il de légitimer les quantités impossibles par leur représentation géométrique, alors qu'il est celui qui introduit à l'Université les travaux d'Adrien-Quentin Buée (1748-1826), de Jean-Robert Argand (1768-1822), de C. V. Mourey, et que John Warren (1796-1852) publie à Cambridge A treatise on the geometrical representation of the square roots of negative quantities (1828)?

- pourquoi n'y a-t-il pas trace d'une présentation axiomatique de l'algèbre symbolique, ni dans le rapport de Peacock, ni dans son traité d'algèbre de 1830 ?

— comment saisir les liens entre les différents acteurs de ce mouvement, liens qui n'apparaissent pas dans les ouvrages précédemment indiqués; le lien avec Herschel donc, jamais cité; le lien avec Babbage, davantage reconnu aujourd'hui comme concepteur d'une « machine analytique » dont les différentes parties correspondent aux mêmes fonctions logiques que celle d'un ordinateur; le lien avec Boole, souvent présenté comme un autodidacte; et le lien avec les auteurs de la seconde génération, qui travaillent davantage sur un calcul des opérations?

- comment comprendre l'émergence de ce mouvement en Angleterre, à partir de Cambridge, à cette époque ? et cela alors que l'enseignement des mathématiques reste attaché à une double fidélité à Newton et à la géométrie euclidienne, et que les travaux qui vont alimenter la réflexion de ces nouveaux algébristes sont d'origine continentale ${ }^{16}$ ?

\section{Contexte d'élaboration et réinterprétation de l'algèbre}

Je voudrais montrer qu'une réponse à toutes ces questions peut être obtenue en ressaisissant cet événement, l'émergence d'une conception symbolique de l'algèbre, là où il s'inscrit dans sa propre histoire ${ }^{17}$, que cette

16. Durand, 1990.

17. VATtimo, 1991. 
conception fait sens là où elle se constitue comme démarche à la fois légitimante et unifiante vis-à-vis des différentes dimensions de cette « réforme » de l'algèbre : dimension conceptuelle, dimension pédagogique et dimension politique, ou institutionnelle, dans lesquelles les membres de cette communauté scientifique se trouvent directement et profondément impliqués. Dimensions qui communiquent entre elles là où s'énoncent les discours qui questionnent les fondements et le rôle de la science au sortir de la Révolution industrielle, dans les manuels, les journaux, les revues, les actes de congrès des sociétés savantes, les correspondances.

Cette étude cherche à prendre en compte le fait que les mathématiques effectuent un travail de symbolisation en même temps qu'elles se constituent dans un contexte où elles proposent un discours de vérité, et que ce discours de vérité, par un effet polysémique inhérent à la langue, rencontre d'autres quêtes de symbolisation de même type, avec lesquelles elles peuvent s'accorder ou entrer en conflit. C'est ainsi qu'elles peuvent se trouver fonctionner tantôt comme discipline isolée, tantôt comme noyau central d'un travail d'élaboration de significations nouvelles qui l'installe non seulement comme référence, mais comme fondement de leur rationalité.

De ce fait, si vérité il y a, elle est multiple dans la mesure même où elle fait sens : le réseau de significations dans lequel elle se trouve insérée au moment de l'élaboration d'une théorie peut être fort différent de celui auquel elle se réfère aujourd'hui. Et, dans la mesure où c'est en tant que production de sens que se trouve examinée l'activité mathématique, ce sont ces différents réseaux de significations, historiquement référés, qu'il convient de restituer. Une telle conception de la pensée mathématique comme production de sens présuppose une activité connaissante qui ne soit pas seulement envisagée comme cognition, mais comme inscription dans un contexte dont les différentes composantes, notamment sociopolitiques, philosophiques, voire théologiques, sont porteuses d'une semblable quête de vérité de leur discours. Elle présuppose aussi un sujet qui déborde la seule référence au cogito cartésien, et qui se construit lui-même au sein du réseau d'interdépendances où prend place sa réflexion.

Plutôt que d'élaboration d'une conception abstraite de l'algèbre, je parlerais plus volontiers ici d'une explicitation du caractère fondamentalement logique de l'opérativité des mathématiques, telles qu'elles s'expriment algébriquement. Reprenant le vocabulaire de Leo Corry ${ }^{18}$, qui distingue entre les contenus des mathématiques et la représentation que s'en donnent les mathématiciens, et qui montre qu'au $\mathrm{xx}^{\mathrm{e}}$ siècle, la représentation des mathématiques en tant qu'étude de structures tarde à se mettre en place

18. CORRY, 1996. 
alors que les contenus sont présents, on dirait qu'ici au contraire se constitue d'abord une nouvelle représentation de la nature des mathématiques, fondée précisément sur le caractère opératoire de l'algèbre, dont il s'agit de légitimer l'instrumentalité par des lois, même si certains contenus restent à préciser. Et cette valeur de légitimation, qui se trouve ainsi conférée au caractère opératoire, est obtenue en séparant l'algèbre de tout recours aux contingences du quantitatif pour mieux l'instituer comme fondement.

\section{LE CONTEXTE DE TRAVAIL DE CE RÉSEAU D’ALGÉBRISTES}

Cette explicitation de la logique opératoire de l'algèbre est issue d'un mouvement de réformes de l'enseignement des mathématiques né à Cambridge en 1812, avec la création de The Analytical Society, qui regroupe une douzaine d'étudiants autour de Babbage. Que signifie ce mouvement? Qu'en est-il de la situation des mathématiques et de leur enseignement à l'Université, en Angleterre à cette époque?

Si l'enseignement des mathématiques constitue alors « la gloire et l'honneur » de l'université de Cambridge, il a lieu dans le cadre institutionnel d'une université anglicane, où les mathématiques participent de ce que John Gascoigne ${ }^{19}$ appelle « l'union sacrée entre science et religion», fondée sur l'enseignement des Principes mathématiques de la philosophie naturelle de Newton, où les mathématiques apparaissent comme le fondement même de l'ordre du monde, un ordre dont le dessein est d'origine divine. Elles ont ainsi à participer, dans leur contenu comme dans leur forme, de l'énonciation de la permanence du monde, comme l'explicitera William Whewell (1794-1866) quelques décennies plus tard ${ }^{20}$. Et c'est bien à cette inscription des mathématiques dans un ensemble de valeurs fondées sur l'attachement au passé, trouvant leur légitimité dans la culture classique et le respect des Anciens, plutôt qu'à la querelle de priorité entre Newton et Leibniz survenue plus d'un siècle plus tôt, qu'il convient d'attribuer le blocage des mathématiques autour des conceptions newtoniennes, et la fidélité à une présentation géométrique des principes du calcul infinitésimal à Cambridge ${ }^{21}$.

Face à cette lourdeur institutionnelle se manifestent dans tout le pays, surtout autour des nouveaux centres industriels, un dynamisme et un intérêt pour la science marqués des valeurs de l'utilitarisme, pour lesquelles toute

19. Gascoigne, 1989, p. 142-184.

20. WhEWELL, 1850.

21. DuRAND-Richard, 1996. 
institution est l'émanation de la société et doit placer son efficacité au service du bonheur du plus grand nombre. Hors de l'université anglicane, dans les académies dissidentes, dans les académies militaires ${ }^{22}$, dans les écoles de commerce et de navigation ${ }^{23}$, le caractère instrumental de la science est valorisé, comme en témoignent dès les années 1808-1810 les interventions du révérend John Playfair (1748-1819), mathématicien et géologiste ${ }^{24}$, dans The Edinburgh Review, revue critique d'inspiration utilitariste créée en 1802 par l'intelligentsia whig. Mais, au sein de l'université anglicane, les mathématiques à Cambridge et la logique à Oxford ont d'abord valeur de fondement des connaissances, et restent les garantes de la constitution de la discipline d'esprit nécessaire aux futurs hommes de loi - pasteurs, avocats, hommes politiques, scientifiques — dont le pouvoir est référé à un ordre du monde. De très nombreuses sociétés provinciales voient le jour, dont la vitalité manifeste un décalage flagrant entre une aristocratie terrienne fidèle à la culture $\mathrm{du} \mathrm{XVIII}^{\mathrm{e}}$ siècle, et les nouvelles élites provinciales en quête d'une légitimation culturelle de leur pouvoir naissant.

Le problème qui se pose au début du XIX ${ }^{\mathrm{e}}$ siècle est celui de l'intégration, dans l'enseignement universitaire, des nouvelles formes de savoir développées sur le Continent ou hors de l'Université, au moment où l'ordre établi se trouve fragilisé par les contradictions sociales issues de la Révolution industrielle anglaise et par l'horizon politique de la Révolution française. L'enjeu des critiques formulées à l'encontre de l'université anglicane est celui de sa transformation, du statut de « seminary of sound learning and religious education » en celui de «national seminary». C'est en ce sens que Playfair prône le développement d'une science utile aux besoins humains et s'attaque aux enseignements traditionnels, arguant du fait que la fidélité à la logique scolastique exclut le recours à l'expérience et à l'observation, tout comme à la curiosité ou à l'esprit d'innovation, c'est-à-dire à toute démarche expérimentale ${ }^{25}$.

C'est autour des réponses à ces critiques que s'articule le double renouvellement des principes de la logique et des mathématiques.

Les réponses des logiciens d'Oxford sont d'abord portées, dès 18081809, par le groupe que Pietro Corsi ${ }^{26}$ nomme «Les Noétiques d'Oxford », qui cherchent notamment à intégrer l'économie politique dans l'enseignement traditionnel, en l'adaptant à l'épistémologie induite par la logique scolastique. Le traité de logique de Richard Whately de 1826, qui servira de manuel de référence à Boole au moment de sa rédaction de Mathemati-

22. Rogers, 1981.

23. Fauvel, Ransom et Wallis, 1991.

24. Playfair, 1808 et 1810.

25. DuRAND-Richard, 1996, p. 454-455.

26. CORSI, 1988. 
cal analysis of logic, est élaboré à cet effet $^{27}$. C'est dans le cadre de ces débats que prend naissance le mouvement de renouveau de la logique que William Hamilton (1788-1856) appellera la « Nouvelle Analytique ${ }^{28}{ }$. La question qui se pose est celle de comprendre si la vérité des assertions produites par des disciplines nouvelles comme l'économie politique provient des données d'un monde en pleine transformation ou du raisonnement luimême. C'est ainsi que le philosophe Dugald Stewart (1753-1828) reprend à son compte la «certitude démonstrative» telle que l'avait conçue John Locke, qui conduit à privilégier la notion de consistance par rapport à celle de vérité puisque, pour les objets mathématiques, existence nominale et existence réelle se trouvent confondues ${ }^{29}$. Whately va plus loin en comparant opérations logiques et opérations mathématiques, insistant dans les deux cas sur l'usage de symboles arbitraires dépourvus de signification. Il distingue entre trois acceptions du terme «opération », que Locke utilisait plus confusément: l'opération comme faculté de l'esprit, l'opération comme mise en œuvre de cette faculté, et l'opération comme résultat. Ses discussions avec Hamilton portent précisément sur l'acceptation pour l'un et le refus pour l'autre du caractère instrumental de la logique, chacun se référant à un niveau différent quant au lieu où se situer pour étudier les processus opératoires : au niveau du raisonnement pour Whately, au niveau du jugement pour Hamilton.

À Cambridge, les initiatives de ce réseau d'algébristes sont d'autant plus remarquables qu'elles sont d'abord le fait d'un groupe d'étudiants issus de milieux sociaux intermédiaires entre ceux qu'il s'agit de réconcilier du côté du pouvoir, et investis d'une audace nouvelle vis-à-vis du savoir traditionnel et de ses institutions. Babbage est fils de banquier, et restera sa vie durant un libéral très proche des radicaux ${ }^{30}$. Herschel est le fils d'un astronome de renommée internationale, mais d'origine modeste et étrangère, qui maintiendra toute sa vie un regard fort distancié envers l'ordre établi. Quant à Peacock, fils d'un vicaire anglican, il s'éloigne assez vite, après son entrée à Cambridge, des idées radicales qu'il partageait alors avec son frère William ${ }^{31}$ pour inscrire délibérément son action institutionnelle ${ }^{32}$ et sa

27. Mais surtout, cette logique est un ouvrage préparé pour l'Encyclopaedia metropolitana de Samuel Taylor Coleridge (1772-1834) qui s'oppose à la spécialisation des sciences, considérée comme une menace de laïcisation et de plébéisation. À cette fin, il en appelle à la constitution d'un corps de théologiens, érudits et hommes de science, afin de préparer un ouvrage offrant, non pas une présentation alphabétique, mais l'exposé rationnel d'un système philosophique fondé sur la nature des sujets abordés, ainsi que sur la genèse et l'harmonie des connaissances humaines. Voir Whately, 1826, et YeO, 1993, p. 44 et 51.

28. Hamilton, 1833.

29. DuRAND, 1990, p. 163-165.

30. Hyman, 1982, p. 38-39, 82-84 et 186.

31. Correspondance : Peacock Mss, Trinity College Cambridge, P $3^{7}, \mathrm{P} 3^{8}, \mathrm{P} 3^{10}$.

32. Peacock est successivement tuteur à Trinity College (1817-1836), examinateur au Senate House Examination (1817, 1819 et 1820), Lowndean Professor (1836-1839). Il publie 
pensée mathématique dans l'histoire de la professionnalisation de l'Université et de la laïcisation de ses statuts.

Faute de pouvoir intervenir directement sur l'Université, dont les institutions datent de 1570 et témoignent bien davantage d'une préoccupation de stabilité que d'adaptation à une société en évolution, leur objectif premier est de faire sortir Cambridge de son provincialisme et la Royal Society de son ronronnement, en créant autour d'elle des sociétés d'importance nationale $^{33}$, ainsi que les journaux qui les accompagnent ${ }^{34}$. Ils sont partie prenante de la création de la British Association of the Advancement of Science en 1831, dont Jack Morrell et Arnold Thackray ont montré que si elle était au départ investie du projet de développer une méthodologie empiriste visant à coordonner les travaux provinciaux, elle s'est assez vite orientée vers une philosophie de la science permettant de soumettre toute démarche empirique au raisonnement déductif ${ }^{35}$. C'est autour de cette conception de la science que le mouvement réformateur cherche à réaliser un nouvel équilibre, visant à dépasser les oppositions entre les «learned men » et les «pratical men », et à faire de la science le catalyseur de l'unification nécessaire à une stabilisation politico-sociale encore incertaine ${ }^{36}$.

PROGRAMME DE RECHERCHE ET THÉORIE DE L'INVENTION

La traduction du traité de Lacroix n'est que le premier acte reconnu d'une volonté politique de réformes, qui dépasse de très loin les quelques tentatives qui ont déjà eu lieu à Cambridge au tournant du siècle pour renforcer la qualité de l'enseignement. Elle est précédée en 1813 par la publication de l'unique volume des Memoirs of the Analytical Society, dont le but explicite est de confronter les mathématiciens de Cambridge aux développements continentaux de l'analyse algébrique. Playfair, dans sa présen-

Observations on the statutes of the university of Cambridge (1841), puis participe à la Commission royale d'enquête (1852) et à la Commission exécutive (1855-1858) qui président à la réforme de l'université de Cambridge de 1858 .

33. Cambridge Philosophical Society en 1819, Royal Astronomical Society en 1820.

34. Transactions of the Cambridge Philosophical Society, puis Cambridge Mathematical Journal,premier journal spécialisé de mathématiques, où s'exprimeront bon nombre des algébristes de la seconde génération.

35. C'est en 1833 à Cambridge que Whewell forge le terme « scientist» pour désigner les participants à cette espèce «d'église nationale de l'intellect », telle que la définit Coleridge, voir Morrell et Thackray, 1981, p. 2-34 et 256-275.

36. Au congrès de la British Association for the Advancement of Science de 1834 à Édimbourg, Dyonisius Lardner investit les scientifiques libéraux de son espoir de les voir élaborer un programme permettant de dépasser les oppositions entre ces deux catégories sociales, voir DURAND-RichARD, 1992, p. 10; LARDNER, 1834. 
tation de la Mécanique céleste de Laplace en 1808, en montrant que la théorie laplacienne, par le biais du calcul leibnizien, confirmait la mécanique newtonienne, avait estompé les préventions contre le caractère instrumental et mécanique des procédures algébriques, et ouvert la voie d'une telle confrontation.

La préface de ce volume fut essentiellement rédigée par Babbage. Outre qu'elle insiste sur l'origine anglaise de bon nombre de ces développements, et sur la nécessité d'en réimporter les acquis ${ }^{37}$, elle présente un véritable programme de recherche d'intérêt national : celui d'introduire et de développer en Angleterre une conception des mathématiques fondée sur l'analyse, qui permette d'unifier les méthodes du calcul des fonctions génératrices de Laplace, le travail de séparation des symboles d'opération de ceux des quantités d'Arbogast, ainsi que les méthodes de résolution des équations différentielles, des équations aux différences finies, des équations aux différences mixtes, et des équations fonctionnelles ${ }^{38}$.

Ce recours à l'analyse ne porte pas uniquement sur le renouvellement des contenus, il concerne également les méthodes. Se référant à Bacon et s'exprimant comme Locke, Babbage conçoit l'écriture algébrique comme un moyen de conduire à une théorie de l'invention qui puisse rompre avec les formes répétitives d'apprentissage de l'enseignement érudit, et s'ouvrir à la recherche :

"Attentively to observe the operations of the mind in the discovery of new truths, and to retain at the same time those fleeting links, which furnish a momentary connection with distant ideas, the knowledge of whose existence we derive from reason rather than from perception, are the objects in whose pursuit nothing but the most patient assiduity can expect success. Powerful indeed, must be the mind, which can simultaneously carry on two processes, each of which requires the most concentrated attention. Yet these obstacles must be surmounted, before we can hope for the discovery of a philosophical theory of invention; a science which Lord Bacon reported to be wholly deficient two centuries ago, and which has made since that time but slight advances ${ }^{39} . »$

Son double mémoire sur le calcul des fonctions, qui apparaît à ses contemporains comme la «véritable métaphysique » des mathématiques ${ }^{40}$ et en fait le fondateur du calcul fonctionnel en tant que domaine spécifique, est construit sur l'idée d'une analogie avec la résolution des équations ordinaires. Et Babbage poursuit, dans les années 1820, une patiente réflexion

37. BabbaGe, 1989, vol. I, p. 37-42.

38. Royal Society, Herschel papers, Hs 2.64, lettre de Herschel à Babbage du 14/07/1816.

39. BabBAGE, 1989, vol. I, p. 59.

40. British Library, Correspondance de Babbage, Add. Mss 37 182, f 155. 
sur ces méthodes d'invention que sont le langage des signes, l'analogie, l'induction et la généralisation, notamment dans un manuscrit resté non publié à ce jour ${ }^{41}$, « The philosophy of analysis », dont il discute abondamment dans sa correspondance avec Peacock.

Cette recherche d'une théorie de l'invention est tout aussi manifeste dans les analyses méthodologiques de Babbage que dans la présentation que donnera Peacock du processus d'explicitation de l'Algèbre Symbolique. D'un point de vue technique, elle puise son inspiration dans la présentation $\mathrm{du}$ «Calcul des fonctions génératrices » qui sert d'introduction à la Théorie analytique du calcul des probabilités. Laplace lui-même y définit ce calcul comme un prolongement des travaux de Viète, Descartes, Wallis, Newton, Leibniz et Lagrange, et le conçoit comme une mise en pratique de l'induction et de l'analogie dans le passage des exposants entiers aux exposants fractionnaires et, plus généralement, dans l'élaboration des méthodes d'interpolation $^{42}$.

L'ALGÈBRE SYMBOLIQUE DE PEACOCK, ISSUE CONCEPTUELLE DE CETTE PERSPECTIVE

Si le travail de Peacock aboutit finalement à une rupture épistémologique en algèbre, ce n'est pas tant en raison d'une volonté initiale de produire une algèbre abstraite, qu'en raison du compromis d'ordre conceptuel qu'il envisage. Entre le souci d'intégrer tous les acquis de l'analyse algébrique, et celui d'échapper aux critiques relatives à l'instrumentalité des pratiques algébriques, il cherche à conférer à l'algèbre la légitimité accordée aux vérités nécessaires, en la séparant de toute référence aux vérités contingentes. Il prend en considération les résultats issus de pratiques inductives ou analogiques qui ne permettent pas de les considérer comme parfaitement fondés en raison, notamment ceux qui traitent des opérations sur les quantités impossibles ou sur l'opérateur différentiel, traitées jusque-là par analogie avec les opérations sur les nombres entiers ou fractionnaires, ou avec des raisonnements géométriques. C'est notamment le cas du théorème de Lagrange, qui donne une écriture finie de la différence d'ordre $\mathrm{n}$ en fonction d'une puissance de l'exponentielle où intervient l'opérateur différentiel, et où la notation d'Arbogast permet d'opérer sur des symboles dépourvus de signification.

Pour légitimer ces acquis, Peacock isole une structure opératoire unique, celle de l'Algèbre Symbolique, obtenue en séparant la logique des opéra-

41. British Library, Babbage papers, Add. Mss 37202.

42. Laplace, 1814 , p. 3-9. 
tions de leur interprétation, et la pose comme logiquement antérieure à toute interprétation, tout en acceptant que ses formes soient suggérées par la pratique.

\section{Le statut de l'Algèbre Symbolique}

C'est en raison même de cette suggestion par l'expérience que l'Algèbre Symbolique n'est pas présentée $a$ priori. Elle intervient comme troisième et dernière étape d'un processus d'abstraction dont l'arithmétique et l'Algèbre Arithmétique constituent les deux premières. L'Algèbre Arithmétique correspond à l'état d'une science algébrique dont la cohérence repose encore sur celle du langage naturel, c'est-à-dire sur le respect des définitions, des pratiques arithmétiques, et donc des limitations inhérentes à leur inscription dans le langage. Peacock insiste sur sa nature algorithmique, grâce à laquelle il peut identifier calculs finis et infinis, polynômes et séries, comme résultats d'un même processus opératoire, celui de la division.

L'Algèbre Symbolique marque donc un renversement radical de point de vue, puisqu'elle est destinée à faire sauter les verrous d'ordre conceptuel issus de l'interprétation attachée à la signification arithmétique. Sa nécessité logique impose l'abandon du sens des symboles et une attention exclusive portée aux propriétés des opérations conçues comme combinaisons de symboles arbitraires. C'est ainsi que Peacock distingue entre égalité arithmétique et équivalence symbolique : le quotient $\mathrm{a} /(1-\mathrm{x})$ et la série $\mathrm{a}+\mathrm{ax}$ $+\mathrm{ax}^{2}+\mathrm{ax}^{3}+\& \mathrm{c}$ sont algébriquement équivalents puisque la série est le résultat opératoire de la division indiquée par le quotient, mais les deux expressions ne sont arithmétiquement égales que si $\mathrm{x}$ est une quantité inférieure à l'unité ${ }^{43}$.

\section{Le principe de permanence des formes équivalentes}

Peacock conçoit tout à fait clairement la nécessité non seulement logique, mais épistémologique, de justifier le saut conceptuel qui sépare alors la pratique arithmétique d'une théorie générale de l'algèbre. Mais dans la mesure où il refuse tout autant de s'appuyer sur une procédure résolument constructive que sur une approche axiomatique, parce que toutes deux sont de nature finie, seul un présupposé ontologique implicite lui permet de légitimer ce saut conceptuel. C'est par le double énoncé du principe

43. РеАСоск, 1830, p. 98-100. 
de permanence des formes équivalentes qu'il s'autorise à transférer à l'Algèbre Symbolique toutes les formes générales obtenues en Algèbre Arithmétique :

«(A): Whatever form is algebraically equivalent to another when expressed in general symbols, must continue to be equivalent, whatever those symbols denote. »

«(B): Converse Proposition: Whatever equivalent form is discoverable in arithmetical algebra considered as the science of suggestion, when the symbols are general in their form, though specific in their value, will continue to be an equivalent form when the symbols are general in their nature as well as their form ${ }^{44}$.»

L'énoncé (B) exprime le fait que la validité de la connaissance acquise réside dans la généralité des formes obtenues. Mais auparavant, l'énoncé (A) suppose de fait la préexistence logique de l'Algèbre Symbolique : comment ces possibilités opératoires générales pourraient-elles être explicitées à partir de la seule expérience sans être conçues comme des découvertes ? Ce double principe sous-tend de fait l'idée d'une finalité préétablie, toute idée neuve étant alors le signe ou l'indice, d'une structure préexistante.

\section{L'épistémologie sous-jacente au travail de Peacock}

Cette conception de l'Algèbre Symbolique relève d'une épistémologie génétique cherchant à respecter l'historicité de la démarche mentale qui préside à son élaboration. Elle est très directement marquée par la conception du langage et de l'abstraction développée par Locke dans son Essay on human understanding, qui appartient au fonds culturel de l'université de Cambridge $^{45}$. Directement inspirée par cette philosophie empiriste, la méthodologie du travail de Peacock, là où elle recourt à l'arithmétique et à l'Algèbre Arithmétique, fait scrupuleusement place à l'étape heuristique qui doit précéder l'énoncé des principes. Conformément au point de vue de Locke sur l'universalité et la vérité, c'est en tant que science spéculative que l'Algèbre Symbolique est universelle, et la vérité y est de l'ordre du langage : elle ne concerne que les possibilités de combinaison des idées et des mots, en tant que signes respectivement naturels et artificiels. Quant à la démonstration, elle consiste à introduire, entre deux idées dont la relation n'est pas immédiate, une suite d'idées intermédiaires appelées preuves entre lesquelles peut s'exercer la connaissance intuitive ${ }^{46}$. Tout comme

44. Peacock, 1833, p. 194.

45. Durand, 1990.

46. LOCKE, 1694, IV.1.9. 
chez Locke lorsqu'il est question de préciser le statut de ce langage ${ }^{47}$, le principe de permanence fait apparaître l'idée de finalité d'un monde conçu comme création achevée. Cette finalité sert à Peacock de légitimation implicite lui permettant d'éviter la rupture épistémologique qui le conduirait à assumer la liberté du mathématicien comme créateur d'un langage formel.

Avec le travail de Peacock, l'objet de la connaissance mathématique change donc de nature : elle s'attache désormais au fonctionnement des opérations considérées pour elles-mêmes, sans plus se préoccuper de la signification attachée aux objets sur lesquels elles travaillent. Cependant, le recours implicite à la finalité de ce langage du raisonnement symbolique suppose, non pas un abandon de toute référence à l'idée de signification, mais un transfert de cette fonction de signification, qui abandonne les objets pour se fixer sur les opérations. Si les symboles sont devenus arbitraires, les opérations restent porteuses de signification dans la mesure même où, comme chez Locke, elles s'articulent implicitement sur les facultés de l'esprit qui les organisent. Peacock affirme ainsi, non seulement l'indépendance de la rationalité de ces opérations vis-à-vis des objets sur lesquels elle s'exerce, mais la primauté ontologique des opérations sur les objets. Si la mise en acte de ces opérations résulte d'une expérience active et volontaire, elle s'appuie sur des facultés qui chez Locke, restent innées. Et l'Algèbre Symbolique est là pour expliciter leurs modes de fonctionnement, leur infinie possibilité d'agir par combinaisons de symboles, identique à elle-même quels que soient les objets manipulés. Ainsi s'éclaire la constance avec laquelle Peacock s'applique à considérer l'Algèbre Symbolique comme une algèbre de l'infini, non pas à proprement parler algèbre des séries formelles, mais algèbre des processus opératoires, ne réalisant qu'à l'infini l'ensemble de ses potentialités.

Quels que soient les présupposés d'ordre ontologique qui sous-tendent la réflexion de Peacock, son travail clôt le débat sur l'indépendance des méthodes de l'analyse algébrique. Elle installe au tout premier plan l'étude globale de ce qui fonde la permanence et l'universalité des formes du calcul algébrique, et relègue au second plan l'étude locale de questions comme la continuité d'une fonction, la convergence d'une série, ou la construction d'un domaine de validité.

Cette séparation radicale entre un registre opératoire fondationnel et un registre interprétatif va se trouver systématiquement reconduite dans les différentes voies de recherche qui vont l'accompagner ou lui succéder, et qui conduiront à un renoncement à l'unicité du registre opératoire.

47. Locke, 1694, IV.4.4, IV.6.14; Duchesneau, 1973, p. 199-202. 
La nouvelle conception des machines qu'édifie Babbage n'est indépendante ni de cette philosophie de l'algèbre dont Peacock affirme le caractère essentiellement symbolique et opératoire, ni de ses propres travaux mathématiques sur le calcul fonctionnel et la notation mathématique, ni de son analyse sociopolitique du système industriel britannique. Son ouvrage intitulé On the economy of machines and manufactures (1831) est en effet une étude encyclopédique entreprise dès 1819 , qui place délibérément et pour la première fois l'usine au centre de la scène économique, prônant l'union de la théorisation scientifique et de la pratique industrielle, et sur laquelle s'appuieront aussi bien John Stuart Mill ${ }^{48}$ que Karl Marx ${ }^{49}$. C'est en spécifiant les conditions et les effets du développement d'une production fondée sur les machines et sur le principe de la division du travail, qu'il est amené, dès la création de la Royal Astronomical Society, à appliquer ce même principe aussi bien au travail mécanique qu'au travail mental, aussi bien à la répartition des travaux d'observation entre les astronomes, qu'à la fabrication des tables astronomiques ${ }^{50}$. Sa démarche, qu'il explicite avant la mathématisation de la logique, relève de ce qu'on appelle aujourd'hui la Seconde Révolution industrielle.

La machine aux différences, dont un premier modèle est construit dès 1822 pour éviter l'intervention humaine dans l'élaboration des tables pour l'astronomie et la navigation, matérialise la séparation entre l'affichage des valeurs numériques et le fonctionnement opératoire qui permet de passer des valeurs d'une différence d'ordre $n$ à celles d'une différence d'ordre $\mathrm{n}-1$, jusqu'à celles de la fonction elle-même. Elle est capable de fournir, à partir d'une suite de valeurs de la variable et des valeurs initiales des différences, la suite des valeurs d'une fonction, dont la forme analytique n'est pas nécessairement connue. Un tel phénomène est alors interprété comme l'existence d'une loi préalable encore inconnue, que le calcul mécanique permet de matérialiser. De fait, la machine aux différences peut traiter toute fonction récursive primitive dont on peut établir la calculabilité par machine $^{51}$.

\section{MiLl, 1848.}

49. MARX, 1950, vol. II, chapitres intitulés : «La division du travail et la manufacture » et « Le machinisme et la grande industrie ».

50. «Address of the Royal Astronomical Society, explanatory of their views and objects » (circulated prior to their first public meeting), Memoirs of the Astronomical Society, 1821, vol. I, p. 1-7.

51. Mosconi, 1983. 
Quant à la machine analytique, dont Babbage élabore les plans à partir de 1834, elle réalise la séparation entre les différentes fonctions opératoires, chacune étant matérialisée par une partie spécifique de la machine : les organes d'entrée-sortie, la mémoire, l'unité de calcul, l'organe de commande, constitué de différents jeux de cartes perforées de type Jacquard et permettant l'exploitation d'un programme externe, et le dispositif de contrôle, dont les cylindres peuvent être assimilés à des sous-ensembles microcodés de programme interne ${ }^{52}$. Autre matérialisation essentielle de cette machine : celle de l'absence de différence de nature entre calculs finis et calculs infinis, puisqu'une telle machine supplée au caractère fini de ses possibilités spatiales, par la possibilité d'une répétition illimitée de ses opérations ${ }^{53}$. Charles Babbage et Luigi F. Menabrea ${ }^{54}$ la reconnaissent comme une machine universelle, pouvant calculer les valeurs de n'importe quelle fonction, là où la machine aux différences n'était qu'une machine particulière traitant d'une suite spécifique d'opérations. Lady Ada Lovelace, fille de lord Byron, amie, confidente et collaboratrice de Babbage, traduit et annote le texte français de Menabrea, donnant le commentaire contemporain le plus approfondi dont on dispose sur la machine analytique. Elle insiste sur le caractère éminemment symbolique des opérations ainsi effectuées, et sur l'adéquation tout à fait remarquable de cette machine avec la méthode de séparation entre symboles d'opération et symboles de quantité, méthode qui inspira la notation d'Arbogast avant de nourrir les recherches du trio Herschel-Peacock-Babbage ${ }^{55}$.

Si elle préfigure un calculateur contrôlé par programme, la machine analytique représente, pour ses contemporains, la matérialisation de la science des opérations. Elle témoigne de la possible matérialisation des lois opératoires et de leur indépendance radicale à l'égard du numérique.

En 1837, le Cambridge Mathematical Journal, fondé par Gregory et Ellis, réalise les ambitions de The Analytical Society. Il se veut un journal de recherche ouvert aux contributions des étudiants ${ }^{56}$, et comporte une

52. DuRAND-RICHARD, 1992

53. BabBage, 1989, vol. XI, p. 93-94.

54. Luigi F. Menabrea, ingénieur et futur premier ministre de l'Italie unifiée, rédige en français, pour la Bibliothèque universelle de Genève, une présentation de la machine analytique après que Babbage en a exposé les principes aux savants italiens réunis par Plana à Turin en 1840, voir Menabrea, 1842.

55. DuRAND-Richard, 1992, p. 29.

56. William Thomson (1824-1907), futur lord Kelvin, et James Sylvester y écriront leurs premiers articles. Voir Smith et Wise, 1989. 
importante section intitulée «Calcul différentiel et calcul aux différences finies », où sont systématiquement explorés les thèmes présentés par Babbage en 1813, et où est utilisé le vocabulaire de Peacock sur les opérations conçues comme combinaisons de symboles, le rôle de suggestion des approches empiristes, et l'équivalence opératoire des formes algébriques, en tant qu'elle est distincte de l'égalité numérique. Loin d'être coupée des recherches de la physique mathématique, portant sur la lumière, le son, la chaleur, le magnétisme et l'électricité, ces articles en reprennent le matériau, issu notamment des travaux de Laplace, Fourier, Poisson et Airy, mais leur ambition est de substituer les méthodes symboliques de résolution à la théorie des fonctions génératrices de Laplace, et de montrer que la séparation des symboles d'opération des symboles de variables est beaucoup plus qu'une manière commode d'exprimer le résultat : les expressions symboliques qu'elle permet d'obtenir sont en elles-mêmes les solutions des équations.

Les difficultés théoriques, liées chez Peacock à l'absence d'un domaine d'étude spécifié a priori et à l'énoncé du double principe de permanence des formes équivalentes, vont conduire à l'éclatement du domaine, et à une prolifération des voies de recherche, dont les orientations vont dépendre de l'aspect de la question investi comme essentiel ${ }^{57}$.

\section{Le calcul des opérations}

C'est dans ce journal que paraissent la plupart des articles sur le « Calcul des opérations », débouchant sur la publication d'ouvrages comme celui de Carmichaël ${ }^{58}$ en 1855 et sur l'enseignement qu'en donnera Morgan à l'université de Londres.

Gregory est le principal animateur de ce courant de recherches. Il radicalise le point de vue de Peacock en considérant cette fois tout symbole de nombre comme celui d'une opération effectuée sur l'unité, conception elle aussi ancrée sur la philosophie de Locke ${ }^{59}$. Comme Peacock, Gregory se démarque des analogies opératoires dont il fait pourtant largement usage, en énonçant un théorème qui se substitue au principe de permanence des formes équivalentes, et devient fondamental comme principe de transfert des propriétés symboliques des opérations :

«Whatever is proved of the latter symbols, from the known laws of their combination, must be equally true of all other symboles which are subject to the same laws of combination ${ }^{60}$.

57. SaLAnSKis, 1997, p. 11.

58. CARMichä̈l, 1855.

59. GREGORY, 1839a, p. 33-35.

60. Gregory, 1839a, p. 34. 
Il développe de cette manière les méthodes symboliques en vue de la résolution des équations différentielles ou aux différences. Elles se retrouvent aujourd'hui dans le calcul opérationnel et en algèbre différentielle.

La loi des indices ou des exposants : $\quad a^{m} \cdot a^{n} x=a^{m+n} x$

la commutativité :

$\mathrm{a}\{\mathrm{b}(\mathrm{x})\}=\mathrm{b}\{\mathrm{a}(\mathrm{x})\}$

la distributivité :

$\mathrm{a}(\mathrm{x})+\mathrm{a}(\mathrm{y})=\mathrm{a}(\mathrm{x}+\mathrm{y})$

sont trois lois opératoires qui, prises ensemble, s'appliquent aussi bien aux nombres, à l'opérateur différentiel, à l'opérateur de déplacement, à l'opérateur de différence finie, et à leurs combinaisons algébriques ${ }^{61}$. Le théorème de transfert permet de leur appliquer le théorème du binôme et de se réapproprier le théorème de Lagrange, rebaptisé forme symbolique du théorème de Taylor. Celui-ci occupe une place d'autant plus centrale qu'il conjugue notation différentielle, identification des puissances et des ordres de différentiation, séparation entre symboles d'opération et de quantité, et qu'il établit un lien structurel entre calcul différentiel et calcul aux différences finies.

C'est précisément l'analyse des propriétés opératoires qui conduit Gregory à envisager l'éclatement de l'Algèbre Symbolique. Définie cette fois comme « la science qui traite de la combinaison des opérations définies par les lois de combinaison auxquelles elles sont soumises », l'Algèbre Symbolique ne constitue plus un édifice unique. Elle s'attache désormais à l'étude spécifique des différentes classes d'opérations obtenues selon les systèmes de lois de combinaison qui les définissent ${ }^{62}$.

Cette étude des lois opératoires pour elles-mêmes, indépendamment de l'interprétation des symboles, s'enracine dans la philosophie mathématique de Peacock et constitue un thème récurrent des travaux de ce réseau d'algébristes. Cette séparation entre calcul symbolique et interprétation ouvre deux possibilités essentielles : versant négatif, celle de rejeter certaines difficultés dans le domaine de l'interprétation, et versant positif, celle d'envisager des opérations non-interprétables.

\section{Propriétés opératoires et structures}

S'ils réinvestissent les apports de recherches mathématiques comme celles d'Augustin Cauchy ou d'Évariste Galois, les auteurs qui explicitent les propriétés des structures mathématiques ou en produisent de nouvelles, continuent à se référer à Peacock, soit explicitement, comme Morgan ou Boole, soit implicitement, comme Cayley, en reconduisant le vocabulaire

61. GREGORY, 1839a, p. 30-31.

62. GRegory, 1840 . 
du calcul symbolique et les lignes de recherche du calcul des opérations. Aussi bien Morgan que Cayley se démarquent par contre de l'approche hamiltonienne de l'algèbre comme science du temps pur ${ }^{63}$.

Morgan, qui publie, de 1842 à 1849, quatre articles intitulés «On the foundation of algebra », se démarque de Stewart et réinvestit la question de la signification des symboles en s'appuyant sur la référence à des états mentaux et à des processus, avant d'analyser la technicité du symbolisme opératoire de l'algèbre. C'est à propos de cette algèbre qu'il appelle désormais «algèbre logique », qu'il énonce les propriétés caractéristiques de ce qu'on nomme aujourd'hui un corps ${ }^{64}$. Il va ensuite hésiter entre l'affirmation de l'existence de plusieurs algèbres, et celle d'une extension de l'algèbre existante. Son analyse des triplets, si elle assume le caractère inventif de la production d'objets nouveaux, repose sur la volonté de préserver la permanence de la « convertibilité » (commutativité) de la multiplication, établie pour les symboles unités grâce auxquels s'expriment les éléments de ces algèbres triples qu'il cherche à constituer ${ }^{65}$. S'il reconnaît l'importance des quaternions de Hamilton ${ }^{66}$, eux aussi inventions mathématiques, il affirme immédiatement que ses triplets en sont strictement indépendants.

Cayley ne se réfère à Peacock ni lorsqu'il énonce les propriétés structurelles d'un groupe ${ }^{67}$, nommé comme tel, ni lorsqu'il étudie le comportement opératoire des éléments de différents groupes finis. C'est pourtant à partir du calcul des opérations qu'il introduit son étude des substitutions. Et il ne manque pas de poursuivre le débat sur la nature de l'algèbre: il reconduit la séparation entre Algèbre Arithmétique et Algèbre Symbolique, devenues logistique et tactique, affirmant plus précisément que l'algèbre tactique inclut les calculs qui relèvent de l'infini dénombrable ${ }^{68}$.

Ainsi, si ces algébristes produisent, comme le souligne Bourbaki, de nouveaux objets mathématiques, c'est d'abord grâce à l'invention de nouvelles lois opératoires, définies à partir de leurs propriétés. Et cette invention, désormais assumée comme telle, ouvre la voie à la possibilité d'une élaboration dialectique entre le calcul symbolique et ses interprétations.

\section{Boole et l'algébrisation de la logique}

Boole est en contact étroit avec les algébristes de la deuxième génération. La préface de sa Mathematical analysis of logic se réfère explicite-

63. DuRAND-Richard, 1995, p. 130-133.

64. Morgan, $1842 \mathrm{~b}$.

65. Morgan, $1849 \mathrm{~b}$.

66. Hamilton, 1844.

67. CAYley, 1854-1859.

68. Cayley, 1864. 
ment à l'Algèbre Symbolique ${ }^{69}$, sans doute plutôt à celle de Gregory qu'à celle de Peacock.

Sa méthode générale d'analyse, qui lui vaut la médaille d' or ${ }^{70}$ de la Royal Society en 1844, prolonge directement cette perspective puisqu'elle applique le calcul des opérations aux équations linéaires à coefficients variables, c'est-à-dire au cas d'opérations non-commutatives. Il maintiendra cet intérêt pour les méthodes symboliques jusque dans ses traités sur les équations différentielles et sur le calcul des différences finies qu'il publiera à la fin des années 1850 , le second étant présenté comme une suite du premier, réitérant ainsi la primauté des calculs sur l'infini vis-à-vis de ceux sur le fini. Boole y réaffirme une conception téléologique de l'histoire, où l'harmonie va de pair avec l'ordre naturel du développement des théories ${ }^{71}$.

Tout comme Peacock, Boole intègre le caractère purement instrumental de la logique en le fondant sur les opérations de l'esprit. Refusant toute démarche métaphysique, il analyse parallèlement les structures syntaxiques du langage naturel et le fonctionnement de l'acte mental de sélection des classes d'objets dans l'entendement ${ }^{72}$. Par ce biais, il dégage ainsi les analogies opératoires entre calcul algébrique et opérations logiques, analogies qui lui permettent d'écrire algébriquement les propositions logiques, en s'appuyant précisément sur le théorème de transfert de Gregory : "If the arithmetical and the logical process are expressed in the same manner, their symbolical expressions will be subject to the same formal law ${ }^{73}$. » $\mathrm{La}$ théorisation booléenne s'articule tout entière sur la séparation entre calcul symbolique et interprétation des symboles, et sur l'idée que le calcul peut porter sur des écritures dépourvues d'interprétation. Elle produit une algèbre spéciale, l'algèbre de la logique, dont les symboles ne représentent que les valeurs 0 et 1 , puisque l'équation fondamentale $\mathrm{x}^{2}=\mathrm{x}$, issue du fait que la réitération de l'opération de sélection donne la même classe d'objets, n'est vérifiée en algèbre que par ces valeurs. C'est de cette équation fondamentale que Boole déduit alors le principe du tiers-exclu, indépendamment de tout psychologisme et de toute métaphysique. Les trois présupposés de l'Algèbre Symbolique, présents chez Boole - le rôle de suggestion des lois opératoires fournies par l'expérience, le caractère nécessaire de ces lois, qui relèvent des opérations de l'esprit, et l'affirmation de leur existence, indépendante de toute interprétation — permettent non seulement d'éclairer ce qui peut apparaitre à un lecteur moderne comme les difficultés de son travail, mais structurent de manière détermi-

69. Boole, 1847 , p. 3.

70. Boole, 1844.

71. BoOle, 1859 , p. v-VI.

72. DuRAND-Richard, 2000.

73. Boole, 1854 , p. 31. 
nante ce premier rapprochement entre mathématique et logique, dépassement radical de la logique aristotélicienne et scolastique ${ }^{74}$.

La problématique dégagée par Peacock, afin de trouver une légitimation des analogies opératoires qui posent problème au début du siècle pour l'intégration des méthodes algébriques au fondement de la connaissance, impulse donc une arborescence de recherches tout à fait fructueuse, marquée par la première rencontre entre algèbre et logique, par leur double légitimation par leur opérativité, par la production de nouveaux objets et par l'élargissement des perspectives opératoires elles-mêmes, lesquelles donneront lieu à la naissance de nouvelles disciplines.

L'unité profonde des travaux de ce réseau d'algébristes anglais de la première moitié $\mathrm{du} \mathrm{XIX}^{\mathrm{e}}$ siècle ne repose pas tant sur la recherche des principes de l'algèbre abstraite, au sens où elle est aujourd'hui définie, que sur la tentative de légitimation de l'instrumentalité de l'algèbre, en un temps où l'instrumentalité en général prend en Angleterre valeur positive, au moins dans les cercles qui encouragent l'institutionnalisation des effets de la Révolution industrielle. Cette tentative débouche sur une double affirmation épistémologiquement capitale : celle, d'une part, de la séparation entre la logique des opérations et les interprétations dont ce calcul peut faire l'objet, et celle, d'autre part, de l'indépendance radicale du symbolisme algébrique. Le caractère suggestif de l'expérience est pris en compte sans être pensé pour autant comme une constructivité possible des mathématiques ou de la logique. Aucun rapport dialectique entre calcul symbolique et interprétation n'est alors systématisé. Les propriétés opératoires restent au contraire le plus souvent référées au fonctionnement de certaines facultés de l'esprit, c'est-à-dire à la croyance à la rationalité de l'esprit humain, dont l'origine divine, qui reste présupposée, garantit le caractère moral de cette instrumentalité.

La distinction qui s'opère en mathématiques au XIX ${ }^{\mathrm{e}}$ siècle entre logique démonstrative et vérité ne résulte donc pas du seul fait de l'émergence des géométries non-euclidiennes, mais également de cette interrogation fondamentale sur la validation de l'automaticité des calculs formels.

C'est dans la mesure où cette séparation est ainsi fondée ontologiquement, et où elle s'accompagne d'une hiérarchie entre logique et interprétation des opérations, qu'elle renvoie le lecteur d'aujourd'hui à un questionnement toujours présent sur les conditions d'une telle séparation. Dès lors que la garantie du divin ne fait plus référence, la question qui se pose aux successeurs de ce mouvement, tant dans le domaine des mathématiques

74. Coumet, 1966, I, p. 3, 7 et 14, II, p. 6-8, et III, p. 8-11. 
que dans celui de la logique, conduit à réinterroger plus avant la légitimité des analogies opératoires et du caractère aveugle de cette instrumentalité du calcul, et à la situer par rapport à ses interprétations, comme le fait aujourd'hui la théorie des modèles. Elle demeure un «tenant de question $^{75} \gg$ pour tous ceux que ne convainc pas l'hypothèse cognitiviste, qui définit l'activité de connaissance comme une computation de représentations symboliques.

Marie-José DuRAND-RICHARD (juin 2000).

75. SALANSKIS, 1997 , p. $10-12$. 


\section{LISTE DES RÉFÉRENCES}

Arbogast (Louis François Antoine), 1800, Du calcul des dérivations, Strasbourg, Levrault Frères.

Babbage (Charles), 1989, The Works of Charles Babbage, éd. Martin CampbellKeLLy, Londres, Pickering, 11 vol.

Boole (George), 1844, "A general method in analysis », Philosophical Transactions, t. CXXXIV, p. 225-282.

Boole (G.), 1847, The Mathematical Analysis of logic, being an essay towards a calculus of deductive reasoning, Cambridge, Macmillan, Barclay \& Macmillan.

Boole (G.), 1854, An investigation on the laws of thought, on which are founded the mathematical theories of logic and probabilities, Londres, repr. in Boole's collected logical papers, Chicago-Londres, 1916, 2 vol., trad. franç. Souleyman Bachir Diagne, Les Lois de la pensée, Paris, Vrin, 1992.

Boole (G.), 1859, A treatise on differential equations, Cambridge, Macmillan and Co.

Boole (G.), 1860, Treatise on the calculus of finite differences, éd. J. F. Moulton, Londres, Macmillan.

Bourbaki (Nicolas), 1969, Éléments d'histoire des mathématiques, Paris, Hermann.

Boyer (Carl B.), 1968, A history of mathematics, New York, Londres, Sidney, Wiley and Sons.

CAnNon (Walter F.), 1964, «Scientists and Broadchurchmen. An early intellectual network », Journal of British Studies, vol. IV, 1, p. 65-88.

CARMichä̈L (Robert), 1855, A treatise on the calculus of operations, designated to facilitate the processes of the differential and integral calculus and the calculus of finite differences, Londres, Longman, Brown, Green and Longmans.

CAYley (Arthur), 1854, "On the theory of groups, as depending on the symbolic equation $\theta^{n}=1 »$, Part I, Philosophical Magazine, vol. VII, p. 40-47, repr. Collected Mathematical Papers, vol. II, p. 123-130. Part II, 1854, Philosophical Magazine, vol. VII, p. 408-409, repr. Collected Mathematical Papers, vol. II, p. 131-132. Part III, 1859, Philosophical Magazine, vol. XVIII, p. 34-37 et 125-126, repr. Collected Mathematical Papers, vol. IV, p. 88-91.

CAYley (A.), 1864, « On the notion and boundaries of algebra », Quarterly Journal of Pure and Applied Mathematics, vol. IV, repr. Collected Mathematical Papers, vol. V, p. 292-294.

CLock (Daniel), 1964, A new approach of algebra, Ph. D. Diss., Madison, University of Wisconsin.

CORRY (Leo), 1996, Modern algebra and the rise of mathematical structures, Bâle, Boston, Berlin, Birkäuser Verlag (Science Networks, Historical Studies).

Corsi (Pietro), 1988, Science and religion. Baden Powell and the Anglican debate, 1800-1860, Cambridge, Cambridge University Press.

Coumet (Ernest), 1966, "Logique, mathématiques et langage dans l'œuvre de G. Boole. I, II et III », Mathématiques et sciences humaines, 15, p. 1-14, 16, p. $1-14$ et 17 , p. 1-12. 
DuChESNEAu (François), 1973, L'Empirisme de Locke, La Haye, M. Nijhoff.

Durand (Marie-José), 1990, « Genèse de l'Algèbre Symbolique en Angleterre. Une influence possible de John Locke», Revue d'histoire des sciences, t. XLIII, 2-3, p. 129-180.

Durand-Richard (Marie-José), 1992, « Charles Babbage (1791-1871). De l'École algébrique anglaise à la "machine analytique" », Mathématiques, informatique et sciences humaines, 118, p. 5-31 et «Erratum », ibid., 120, p. 79-82.

DurAND-Richard (M.-J.), 1995, «L'impact des travaux de l'École algébrique anglaise dans les journaux scientifiques autour de $1830 »$, Rivista di Storia della Scienza, sér. II, vol. III, 2, p. 119-156.

DurAND-Richard (M.-J.), 1996, «L'École algébrique anglaise. Les conditions conceptuelles et institutionnelles d'un calcul symbolique comme fondement de la connaissance », in Catherine Goldstein, Jeremy GRAY et Jim RitTER, éd., L'Europe mathématique. Histoires, mythes, identités, Paris, Éd. de la Maison des sciences de l'homme, p. 445-477.

DURAND-RICHARD (M.-J.), 2000, « Logic versus algèbre. English debates and Boole's mediation », in James Gasser, éd., Anthology on Boole, Dordrecht, Kluwer Academic Publ. (Synthese Library).

Fauvel (John), Ransom (Peter) et Wallis (Peter et Ruth), 1991, Mathematical tradition in the north of England, Durham, NEBMA, p. 16-19 et 27-32.

Gascoigne (John), 1989, Cambridge in the age of the Enlightenment. Science, religion and politics from the Restoration to the French Revolution, Cambridge, Cambridge University Press.

Graves (Robert P.), 1882, 1885, 1889 et 1891, Life of sir William Rowan Hamilton, including selections from his poems, correspondence, and miscellaneous writings, Londres, Longmans, Green \& Co., 3 vol. et add.

Gregory (Duncan F.), 1839a, " On the solution of linear differential equations with constant coefficients », Cambridge Mathematical Journal, vol. I, p. 25-36, repr. in Mathematical Writings, Cambridge, 1865, p. 14-27.

Gregory (D. F.), 1839b, «Demonstrations of theorems in the differential calculus and calculus of finite differences ", Cambridge Mathematical Journal, vol. I, p. 232-244, repr. in Mathematical Writings, Cambridge, 1865, p. 108-123.

Gregory (D. F.), 1840, "On the real nature of Symbolical Algebra », Transactions of the Royal Society of Edinburgh, vol. XIV, p. 208-216.

Hamilton (William), 1833, «Recent publications on logical science », Edinburgh Review, vol. LVIII, 115, p. 194-238, repr. in Discussions on philosophy and litterature, education and University reform, chiefly from Edinburgh Review, Londres, 1852, p. 116-174.

Hamilton (William Rowan), 1931, 1940 et 1967, The Mathematical Papers of sir William Rowan Hamilton. Vol. I : Geometrical optics, éd. Arthur William Conway et John Lighton Synge; vol. II : Dynamics, éd. A. W. Conway et J. L. Synge; vol. III : Algebra, éd. Heini Halberstam et R. E. Ingram, Cambridge, Cambridge University Press.

Hamilton (W. R.), 1837, «Theory of conjugate functions, or algebraic couples; with a preliminary and elementary essay on algebra as the science of pure time », Transactions of the Royal Irish Academy, vol. XVII, p. 203-422, repr. in Mathematical Papers, Cambridge, 4 vol., 1967, vol. III, p. 4-96.

Hamilton (W. R.), 1844, « On a new species of imaginary quantities connected with the theory of quaternions », Proceedings of the Royal Irish Academy, vol. II, p. 424-434, repr. in Mathematical Papers, Cambridge, 4 vol., 1931, vol. III, p. 414 . 
Hamilton (W. R.), 1848, « Researches respecting quaternions. First Series », Transactions of the Royal Irish Academy, vol. XXI, p. 199-296 [communiqué le 13 nov. 1843], repr. in Mathematical Papers, Cambridge, 4 vol., 1931, vol. III, p. $159-226$.

Hamilton (W. R.), 1853, Lectures on quaternions, Dublin.

Hankins (Thomas L.), 1980, Sir William Rowan Hamilton, Baltimore, Londres, The Johns Hopkins University Press.

Hyman (Anthony), 1982, Charles Babbage, pioneer of the computer, Princeton, NJ, Princeton University Press.

Kelland (Philip), 1840, « On general differentiation. I », Transactions of the Royal Society Edinburgh, vol. XIV, p. 567-603.

KuINE (Morris), 1972, Mathematical thought from ancient to modern times, New York, Oxford University Press.

Koppelman (Elaine H.), 1969, Calculus of operations. French influence on the British mathematics in the first half of the nineteenth century, Ph. D. Diss., Baltimore, MD, Johns Hopkins University.

Laplace (Pierre Simon de), 1814, Théorie analytique des probabilités, $2^{\mathrm{e}}$ éd. Paris.

LARDNER (Dyonisius), 1834, «Babbage's calculating engine », Edinburgh Review, vol. LIX, p. 263-327, repr. in BABBAGE, 1989, vol. II, p. 118-186.

Locke (John), 1694, An essay concerning human understanding, $2^{\mathrm{e}}$ éd. Londres, Essai philosophique concernant l'entendement humain, trad. franç. de l'anglais par M. Coste, $1700,5^{\mathrm{e}}$ éd. Paris, 1755, rééd. Paris, Vrin, 1983.

MarX (Karl), 1950, Le Capital, Paris, Éditions sociales, vol. II.

Menabrea (Luigi F.), 1842, « Notions sur la machine analytique de M. Charles Babbage », Bibliothèque universelle de Genève, t. XLI, 82, repr. in BABBAGE, 1989, vol. III.

Mill (John Stuart), 1848, Principles of political economy, Londres, W. J. Ashley.

Morgan (Augustus de), 1842a, « On the foundations of algebra. I», Transactions of the Cambridge Philosophical Society, 1837-1842, vol. VII, p. 173-187.

Morgan (A. de), 1842b, « On the foundations of Algebra. II », Transactions of the Cambridge Philosophical Society, 1837-1842, vol. VII, p. 287-300.

Morgan (A. de), 1849a, "On the foundations of Algebra. III », Transactions of the Cambridge Philosophical Society, 1844-1849, vol. VIII, p. 139-142.

Morgan (A. de), 1849b, «On the foundations of Algebra. IV, On Triple Algebra », Transactions of the Cambridge Philosophical Society, 1844-1849, vol. VIII, p. 241-254.

Morrel (Jack) et Thackray (Arnold), 1981, Gentlemen of science. Early years of the British Association for the Advancement of Science, Oxford, Clarendon Press.

Mosconi (Jean), 1983, «Charles Babbage. Vers une théorie du calcul mécanique », Revue d'histoire des sciences, t. XXXVI, 1, p. 69-107.

Novy (Lubos), 1968, "L'École algébrique anglaise », Revue de synthèse, III ${ }^{\mathrm{e}}$ sér., t. LXXXIV, 49-52, janv.-déc., p. 211-222.

Peacock (George), (1826) 1845, «Arithmetic », Encyclopaedia metropolitana, Londres, vol. I, p. 369-523.

Ренсоск (G.), 1830, Treatise of Algebra, $1^{\text {re }}$ éd. Cambridge, rééd. Cambridge, 18421845,2 vol.

Peacock (G.), 1833, «A report on the recent progress and present state of certain branches of analysis », Report of the British Association for the Advancement of Science, vol. III, p. 185-351.

Peacock (G.), 1841, Observations on the statutes of the university of Cambridge, Cambridge. 
Playfair (John), [Anonymous], 1808, «Review of Laplace's Traité de mécanique céleste », The Edinburgh Review, vol. XI, 22, p. 249-284.

Playfair (J.), [Anonymous], 1810, Review : «A reply to the calumnies of the Edinburgh Review against Oxford; containing an account of studies pursued in that University », The Edinburgh Review, vol. XVI, 31, p. 158-187.

Rogers (Leo), 1981, «A survey of factors affecting the teaching of mathematics outside the universities in Britain in the nineteenth century », in Herbert MEHRTENs, Henk Bos et Ivo SchneIDER, éd., Social history of mathematics, Boston, Birkhäuser, p. 149-164.

Salanskis (Jean-Michel), 1997, Le Temps du sens, Orléans, HYX.

Sмiтн (Charles) et Wise (M. Norton), 1989, Energy and empire. A biographical study of lord Kelvin, Cambridge, Cambridge University Press.

Vattimo (Gianni), 1991, Éthique de l'interprétation, Paris, La Découverte.

Whately (Richard), 1826, Elements of logic, Londres, repr. in Encyclopaedia metropolitana, Londres, 1849.

Whewell (William), 1850, On a liberal education in general, and with particular reference to the leading studies in the university of Cambridge, $1^{\mathrm{re}}$ éd. 1845, ici Londres, J. W. Parker.

Yeo (Richard), 1993, Defining science. William Whewell, natural knowledge and public debate in early Victorian Britain, Cambridge, Cambridge University Press. 